\title{
Gravitational form factors of $\rho$ meson with a light-cone constituent quark model
}

\author{
Bao-Dong Sun $\odot^{1-3, *}$ and Yu-Bing Dong ${ }^{2-4, \dagger}$ \\ ${ }^{1}$ Key Laboratory of Particle Physics and Particle Irradiation, Ministry of Education, \\ Institute of Frontier and Interdisciplinary Science, Shandong University, Shandong 266237, China \\ ${ }^{2}$ Institute of High Energy Physics, Chinese Academy of Sciences, Beijing 100049, \\ People's Republic of China \\ ${ }^{3}$ School of Physics, University of Chinese Academy of Sciences, Beijing 100049, \\ People's Republic of China \\ ${ }^{4}$ Theoretical Physics Center for Science Facilities (TPCSF), CAS, Beijing 100049, \\ People's Republic of China
}

(Received 17 February 2020; accepted 2 May 2020; published 14 May 2020)

\begin{abstract}
The $\rho$ meson gravitational form factors are studied based on a light-front constituent quark model which has been successfully employed to calculate its generalized parton distributions and some lowenergy observables. The distributions of energy, spin, pressures, and shear forces inside the $\rho$ meson are explicitly given.
\end{abstract}

DOI: 10.1103/PhysRevD.101.096008

\section{INTRODUCTION}

We know that the gravitational form factors (GFFs) are defined through the matrix element of the energymomentum tensor (EMT) [1]. Since the GFFs relate to the mass, spin, shear forces, and $D$-term of the particles [2,3], they (or EMT form factors) involve a large range of physics, such as the gravitation physics and the physics in hard scattering processes $[4,5]$. It is a promising way to extract more information about the mechanical properties of a hadron (especially in the nonperturbative region) from the study of GFFs. Those tasks mainly try to answer some fundamental questions, like how the hadron mass and spin are carried out by quarks and gluons or what the mechanism that the trace anomaly contributes to hadron mass is, and how the strong force distributes inside the hadron, etc.. Besides, the Fourier transforms of the EMT matrix elements define the static EMT which can further tell the distributions of pressure and shear forces [4,5].

In the 1960s, the total GFFs were already introduced for both spin- 0 and spin- $1 / 2$ hadrons [1]. The most natural but also the least practical way to probe GFFs is scattering processes through graviton exchange. However, it's more practical to extract GFFs through their connections to the

\footnotetext{
sunbd@sdu.edu.cn

†dongyb@ihep.ac.cn
}

Published by the American Physical Society under the terms of the Creative Commons Attribution 4.0 International license. Further distribution of this work must maintain attribution to the author(s) and the published article's title, journal citation, and DOI. Funded by SCOAP . generalized parton distributions (GPDs). The relations between GFFs and GPDs were discussed in detail in Refs. [6,7]. As the soft part of the hard-exclusive reactions, GPDs have been received many theoretical and experimental investigations [8-13]. As a reflection of the broken scale invariance of QCD, the matrix element of the trace anomaly part of EMT naturally connects with the hadron mass $[14,15]$. Especially the gluonic operator is believed to contribute to the majority part. This may give another possible way to probe the GFFs via the exclusive production of heavy quarkonium states, such as near-threshold $J / \psi$ and $\Upsilon$ photoproduction processes at JLab. and RHIC etc. [16-18].

At present, it is still not clear what the specific relations among the strong force, pressure, and shear forces are. Nevertheless, one may get some hints from the phenomenological studies of the static EMT of particles with different spins. For instance, the GFFs of pion (spin-0) were evaluated in chiral quark models in Refs. $[19,20]$ and parameter methods [21]. Reference [22] applies the Q-ball model to spin- 0 particles as well, where the $D$-term, energy density, pressure, and shear forces were investigated in detail. There are also lattice QCD calculations related to the pion GFFs [23]. For the spin- $1 / 2$ hadrons, there are model calculations from the AdS/QCD approach [24], and the chiral quark soliton model [25], etc.. More can be found in a review article (see Ref. [4]). The formalism of GFFs for a spin-1 hadron are discussed by Refs. [26-28] and for arbitrary spin hadrons in recent Ref. [29]. In the literature, the model calculations for the spin-1 particles include the AdS/QCD approach [30] and the Nambu-Jona-Lasinio (NJL) model [31]. 
It is shown that the light-cone quark model (LCCQM) for the $\rho$ meson employed in our previous works can describe the $\rho$ meson well in the low energy region, such as its electromagnetic form factors, GPDs, etc. [32-34]. In this work, we'll apply our LCCQM and the previous results of GPDs to study the $\rho$ meson GFFs and its mechanical properties (quadrupole pressure and shear forces, etc. $[27,35])$, and try to get some information about those fundamental questions.

This paper is organized as follows. In Sec. II, the definitions of GFFs, pressure, and shear forces for a spin-1 particle are briefly presented. Moreover, the LCCQM for the $\rho$ meson applied in our previous works is also shortly reviewed in this section. Section III gives our numerical results for the $\rho$ meson GFFs, pressure, and shear forces, etc.. In this part, we introduce a phenomenological three-dimensional (3D) Gaussian form wave package, when calculating the static EMT, since our obtained
GFFs do not drop fast enough. We also display our model-dependent $D$-term of the $\rho$ meson, which is not affected by the Gaussian form wave package. Finally, Sec. IV is devoted for a summary.

\section{GFFS OF SPIN ONE PARTICLES AND OUR MODEL}

\section{A. GFFs of spin one particles}

The formalism of GFFs of a spin one particle and its other mechanical properties have been discussed and given explicitly [26-28]. Here, we briefly summarize them as follows. In this paper, we use the covariant normalization $\left\langle p^{\prime}, \sigma^{\prime} \mid p, \sigma\right\rangle=2 p^{0}(2 \pi)^{3} \delta^{(3)}\left(\vec{p}^{\prime}-\vec{p}\right) \delta_{\sigma \sigma^{\prime}}$ for the system, and introduce the kinematic variables $P=\frac{1}{2}\left(p^{\prime}+p\right)$, $\Delta=p^{\prime}-p, t=\Delta^{2}$. Then, the symmetric (Belinfante) EMT form factors of a spin-1 particle in QCD are defined as,

$$
\begin{aligned}
\left\langle p^{\prime}, \sigma^{\prime}\left|\hat{T}_{\mu \nu}^{a}(x)\right| p, \sigma\right\rangle= & {\left[2 P_{\mu} P_{\nu}\left(-\epsilon^{\prime *} \cdot \epsilon A_{0}^{a}(t)+\frac{\epsilon^{\prime *} \cdot P \epsilon \cdot P}{m^{2}} A_{1}^{a}(t)\right)+2\left[P_{\mu}\left(\epsilon_{\nu}^{\prime *} \epsilon \cdot P+\epsilon_{\nu} \epsilon^{* *} \cdot P\right)+P_{\nu}\left(\epsilon_{\mu}^{\prime *} \epsilon \cdot P+\epsilon_{\mu} \epsilon^{\prime *} \cdot P\right)\right] J^{a}(t)\right.} \\
& +\frac{1}{2}\left(\Delta_{\mu} \Delta_{\nu}-g_{\mu \nu} \Delta^{2}\right)\left(\epsilon^{\prime *} \cdot \epsilon D_{0}^{a}(t)+\frac{\epsilon^{\prime *} \cdot P \epsilon \cdot P}{m^{2}} D_{1}^{a}(t)\right)+\left[\frac{1}{2}\left(\epsilon_{\mu} \epsilon_{\nu}^{\prime *}+\epsilon_{\mu}^{\prime *} \epsilon_{\nu}\right) \Delta^{2}-\left(\epsilon_{\mu}^{\prime *} \Delta_{\nu}+\epsilon_{\nu}^{\prime *} \Delta_{\mu}\right) \epsilon \cdot P\right. \\
& \left.+\left(\epsilon_{\mu} \Delta_{\nu}+\epsilon_{\nu} \Delta_{\mu}\right) \epsilon^{\prime *} \cdot P-4 g_{\mu \nu} \epsilon^{\prime *} \cdot P \epsilon \cdot P\right] E^{a}(t)+\left(\epsilon_{\mu} \epsilon_{\nu}^{\prime *}+\epsilon_{\mu}^{\prime *} \epsilon_{\nu}-\frac{\epsilon^{\prime *} \cdot \epsilon}{2} g_{\mu \nu}\right) m^{2} \bar{f}^{a}(t) \\
& \left.+g_{\mu \nu}\left(\epsilon^{\prime *} \cdot \epsilon m^{2} \bar{c}_{0}^{a}(t)+\epsilon^{\prime *} \cdot P \epsilon \cdot P \bar{c}_{1}^{a}(t)\right)\right] e^{i\left(p^{\prime}-p\right) x}
\end{aligned}
$$

where $m$ is $\rho$ meson mass and $a=g, u, d, \ldots$, which represent the contributions of gluon and all flavors of quarks, and the polarization vectors $\epsilon_{\mu}^{\prime}=\epsilon_{\mu}\left(p^{\prime}, \sigma^{\prime}\right), \epsilon_{\mu}=$ $\epsilon_{\mu}(p, \sigma)$ with $\sigma=x, y, z$, respectively. The 6 quark and gluon GFFs $A_{0,1}^{a}, D_{0,1}^{a}, J^{a}$ and $E^{a}(t)$ are individually momentum-energy conserving, and the other 3 GFFs, $\bar{f}^{a}$ and $\bar{c}_{0,1}^{a}(t)$, are not.

As shown in our previous works, in the Breit frame, the above expression can be reorganized according to the power of the quadrupole operator of the spin one particles. The static EMT $T^{\mu \nu}\left(\vec{r}, \sigma^{\prime}, \sigma\right)$ of the spin-1 system is defined by the Fourier transform of the EMT with respect to $\vec{\Delta}$ as

$T_{a}^{\mu \nu}\left(\vec{r}, \sigma^{\prime}, \sigma\right)=\int \frac{d^{3} \Delta}{2 E(2 \pi)^{3}} e^{-i \vec{\Delta} \cdot \vec{r}}\left\langle p^{\prime}, \sigma^{\prime}\left|\hat{T}_{a}^{\mu \nu}(0)\right| p, \sigma\right\rangle$.

Equation (2) contains the energy densities, the distributions of spin, pressure and shear forces with different power of quadrupole operator. For the energy distributions, we have (sum over all gluons and quark flavors)

$$
\begin{aligned}
T^{00}\left(\vec{r}, \sigma^{\prime}, \sigma\right) & =\int \frac{d^{3} \Delta}{2 E(2 \pi)^{3}} e^{-i \vec{\Delta} \cdot \vec{r}}\left\langle p^{\prime}, \sigma^{\prime}\left|\hat{T}^{00}(0)\right| p, \sigma\right\rangle \\
& =\varepsilon_{0}(r) \delta_{\sigma^{\prime} \sigma}+\varepsilon_{2}(r) \hat{Q}^{i j} Y_{2}^{i j},
\end{aligned}
$$

where $r=|\vec{r}|, Y_{2}^{i j}=r^{i} r^{j} / r^{2}-\delta^{i j} / 3, \hat{Q}^{i j}=\left(\hat{Q}^{i j}\right)_{\sigma^{\prime} \sigma}$, and

$$
\begin{gathered}
\varepsilon_{0}(r)=m \tilde{\mathcal{E}}_{0}(r), \\
\varepsilon_{2}(r)=-\frac{1}{2 m} r \frac{d}{d r} \frac{1}{r} \frac{d}{d r} \tilde{\mathcal{E}}_{2}(r),
\end{gathered}
$$

with

$$
\tilde{\mathcal{E}}_{0,2}(r)=2 m \int \frac{d^{3} \Delta}{2 E(2 \pi)^{3}} e^{-i \vec{\Delta} \cdot \vec{r}} \mathcal{E}_{0,2}(t)
$$

where $\mathcal{E}_{0,2}(t)=\sum_{a} \mathcal{E}_{0,2}^{a}(t)$ and, in the Breit frame, $t=$ $-\vec{\Delta}^{2}$ and $E=\sqrt{m^{2}+\vec{\Delta}^{2} / 4}$. 
For the spin distribution, the $0 j$ component is

$T_{a}^{0 j}\left(\vec{r}, \sigma^{\prime}, \sigma\right)=\int \frac{d^{3} \Delta}{2 E(2 \pi)^{3}} e^{-i \vec{\Delta} \cdot \vec{r}}\left\langle p^{\prime}, \sigma^{\prime}\left|\hat{T}_{a}^{0 j}(0)\right| p, \sigma\right\rangle$

The individual contributions of quarks and gluons to the spin of the particle is

$$
\begin{aligned}
J_{a}^{i}\left(\vec{r}, \sigma^{\prime}, \sigma\right)= & \epsilon^{i j k} r^{j} T_{a}^{0 k}\left(\vec{r}, \sigma^{\prime}, \sigma\right), \\
= & \hat{S}_{\sigma^{\prime} \sigma}^{j} \int \frac{d^{3} \Delta}{(2 \pi)^{3}} e^{-i \vec{\Delta} \cdot \vec{r}}\left[\left(\overline{\mathcal{J}}^{a}(t)+\frac{2}{3} t \frac{d \overline{\mathcal{J}}^{a}(t)}{d t}\right) \delta^{i j}\right. \\
& \left.+\left(\Delta^{i} \Delta^{j}-\frac{1}{3} \vec{\Delta}^{2} \delta^{i j}\right) \frac{d \overline{\mathcal{J}}^{a}(t)}{d t}\right],
\end{aligned}
$$

with $\overline{\mathcal{J}}^{a}(t)=\frac{m}{E} \mathcal{J}^{a}(t)$, and the spin operator [27,36]. ${ }^{1}$

$$
\hat{S}_{\sigma^{\prime} \sigma}^{i}=-i \epsilon^{i j k} \epsilon_{\sigma}^{* j} \epsilon_{\sigma^{\prime}}^{k},\left(i, j, k, \sigma^{\prime}, \sigma=x, y, z\right),
$$

where the rest frame spin-1 polarization vectors are

$$
\epsilon_{x}=\left(\begin{array}{l}
1 \\
0 \\
0
\end{array}\right), \quad \epsilon_{y}=\left(\begin{array}{l}
0 \\
1 \\
0
\end{array}\right), \quad \epsilon_{z}=\left(\begin{array}{l}
0 \\
0 \\
1
\end{array}\right)
$$

For the $i j$-components, the quadrupole elastic pressure and shear forces are firstly defined in Ref. [27] in the sprit of Ref. [37-39]. A new parametrization of pressure and shear forces is introduced in a recent paper [35], and it conveniently generates the normal and tangential forces acting on radial area element $\left(d F_{r}, d F_{\theta}\right.$, and $\left.d F_{\phi}\right)$. These forces explains how the hadron shape forms. The corresponding relations of two sets of parametrization are given in the Appendix of Ref. [35]. According to Ref. [35],

$$
\begin{aligned}
T^{i j}(\vec{r})= & \int \frac{d^{3} \Delta}{2 E(2 \pi)^{3}} e^{-i \vec{\Delta} \cdot \vec{r}}\left\langle p^{\prime}, \sigma^{\prime}\left|\hat{T}^{i j}(0)\right| p, \sigma\right\rangle \\
= & p_{0}(r) \delta^{i j}+s_{0}(r) Y_{2}^{i j}+\left(p_{2}(r)+\frac{1}{3} p_{3}(r)-\frac{1}{9} s_{3}(r)\right) \hat{Q}^{i j} \\
& +\left(s_{2}(r)-\frac{1}{2} p_{3}(r)+\frac{1}{6} s_{3}(r)\right) 2\left[\hat{Q}^{i p} Y_{2}^{p j}+\hat{Q}^{j p} Y_{2}^{p i}-\delta^{i j} \hat{Q}^{p q} Y_{2}^{p q}\right] \\
& +\hat{Q}^{p q} Y_{2}^{p q}\left[\left(\frac{2}{3} p_{3}(r)+\frac{1}{9} s_{3}(r)\right) \delta^{i j}+\left(\frac{1}{2} p_{3}(r)+\frac{5}{6} s_{3}(r)\right) Y_{2}^{i j}\right]+\ldots
\end{aligned}
$$

where the quadrupole pressure $p_{n}(r)$ and shear forces functions $s_{n}(r)$ can be written as

$$
\begin{gathered}
p_{n}(r)=\frac{1}{6 m} \partial^{2} \tilde{\mathcal{D}}_{n}(r)=\frac{1}{6 m} \frac{1}{r^{2}} \frac{d}{d r} r^{2} \frac{d}{d r} \tilde{\mathcal{D}}_{n}(r), \\
s_{n}(r)=-\frac{1}{4 m} r \frac{d}{d r} \frac{1}{r} \frac{d}{d r} \tilde{\mathcal{D}}_{n}(r),
\end{gathered}
$$

and we found,

$$
\begin{gathered}
\tilde{\mathcal{D}}_{0}(r)=2 m \int \frac{d^{3} \Delta}{2 E(2 \pi)^{3}} e^{-i \vec{\Delta} \cdot \vec{r}} \mathcal{D}_{0}(t) \\
\tilde{\mathcal{D}}_{2}(r)=2 m \int \frac{d^{3} \Delta}{2 E(2 \pi)^{3}} e^{-i \vec{\Delta} \cdot \vec{r}} \mathcal{D}_{2}(t)+\frac{2}{m}\left(\frac{d}{d r} \frac{d}{d r}-\frac{2}{r} \frac{d}{d r}\right) \int \frac{d^{3} \Delta}{2 E(2 \pi)^{3}} e^{-i \vec{\Delta} \cdot \vec{r}} \mathcal{D}_{3}(t), \\
\tilde{\mathcal{D}}_{3}(r)=-\frac{4}{m}\left(\frac{d}{d r} \frac{d}{d r}-\frac{2}{r} \frac{d}{d r}\right) \int \frac{d^{3} \Delta}{2 E(2 \pi)^{3}} e^{-i \vec{\Delta} \cdot \vec{r}} \mathcal{D}_{3}(t)
\end{gathered}
$$

\footnotetext{
${ }^{1}$ In Ref. [27], the spin operator and rest frame spin-1 polarization vectors in Eq. (13) and (14) are incorrect.
} 
with $\mathcal{D}_{n}(t)=\sum_{a} \mathcal{D}_{n}^{a}(t)$. The detailed definitions for the form factors $\mathcal{E}_{0,2}^{a}(t), \mathcal{J}^{a}(t)$, and $\mathcal{D}_{0,2,3}^{a}(t)$ are shown in the Appendix A. The spherical components of the force $\left(d F_{r}\right.$, $d F_{\theta}$, and $d F_{\phi}$ ) acting on the infinitesimal radial area element $d S_{r}\left(d \boldsymbol{S}=d S_{r} \boldsymbol{e}_{r}+d S_{\theta} \boldsymbol{e}_{\theta}+d S_{\phi} \boldsymbol{e}_{\phi}\right)$ are given in Ref. [35]. For completeness, we include the results in Appendix B. As shown in Eq. (B1) and (B2), in the unpolarized case, only the normal force $d F_{r} / d S_{r}$ exists inside the particle system and it only has contributions from $p_{0}(r)$ and $s_{0}(r)$. In polarized cases, both normal and tangential forces shows up, together with the higherordered ones, $p_{2}(r), s_{2}(r), p_{3}(r)$, and $s_{3}(r)$.

\section{B. Phenomenological light-front constituent quark}

We know that the GFFs can be obtained from GPDs via the sum rules. For the quark sector, one has

$$
\begin{gathered}
\int_{-1}^{1} x d x H_{1}^{q}(x, \xi, t)=A_{0}^{q}(t)-\xi^{2} D_{0}^{q}(t)+\frac{t}{6 m^{2}} E^{q}(t)+\frac{1}{3} \bar{f}^{q}(t), \\
\int_{-1}^{1} x d x H_{2}^{q}(x, \xi, t)=2 J^{q}(t), \\
\int_{-1}^{1} x d x H_{3}^{q}(x, \xi, t)=-\frac{1}{2}\left[A_{1}^{q}(t)+\xi^{2} D_{1}^{q}(t)\right], \\
\int_{-1}^{1} x d x H_{4}^{q}(x, \xi, t)=-2 \xi E^{q}(t), \\
\int_{-1}^{1} x d x H_{5}^{q}(x, \xi, t)=\frac{t}{2 m^{2}} E^{q}(t)+\bar{f}^{q}(t),
\end{gathered}
$$

and they are similar to the gluon ones. [27,28]. Figure 1 illustrates the process we are considering for GPDs in our phenomenological model. The notations are [32]

$$
\begin{aligned}
& t=\Delta^{2}=\left(p^{\prime}-p\right)^{2}=\left(q-q^{\prime}\right)^{2}, \quad Q^{2}=-q^{2}, \\
& \xi=-\frac{\Delta \cdot n}{2 P \cdot n}=-\frac{\Delta^{+}}{2 P^{+}}, \quad|\xi|=\frac{\Delta^{+}}{2 P^{+}}, \quad(|\xi| \leq 1) \\
& x=\frac{k \cdot n}{P \cdot n}=\frac{k^{+}}{P^{+}}, \quad(-1 \leq x \leq 1),
\end{aligned}
$$

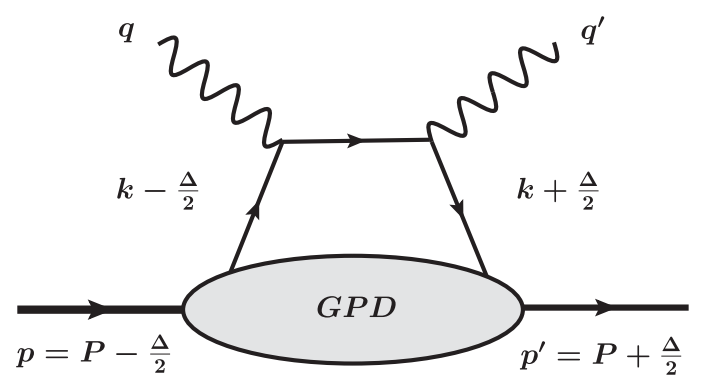

FIG. 1. The s-channel handbag diagram for GPDs. The u-channel one can be obtained by $q \leftrightarrow q^{\prime}$. where $n$ is a lightlike 4-vector. Here $q$ is the virtual photon momentum, and $q^{\prime}$ is treated as a real one.

In a numerical calculation, we employ the phenomenological light-front quark model to describe the interaction between the spin- $1 \rho$ meson and its two constitutes $u$ and $d$ $[32,40]$. It is based on an effective interaction Lagrangian for the $\rho \rightarrow \bar{q} q$ vertex,

$$
\begin{aligned}
\mathcal{L}_{I} & =-\frac{i m_{q}}{f_{\rho}} \bar{q} \Gamma^{\mu} \tau q \cdot \boldsymbol{\rho}_{\mu} \\
& =-\frac{i \sqrt{2} m_{q}}{f_{\rho}}\left[\frac{\bar{u} \Gamma^{\mu} u-\bar{d} \Gamma^{\mu} d}{\sqrt{2}} \rho_{\mu}^{0}+\bar{u} \Gamma^{\mu} d \rho_{\mu}^{+}+\bar{d} \Gamma^{\mu} u \rho_{\mu}^{-}\right],
\end{aligned}
$$

where $\rho_{\mu}$ is the $\rho$ meson field, $f_{\rho}$ is the $\rho$ decay constant, and $\Gamma^{\mu}$ is a Bethe-Salpeter amplitude (BSA) describing the interaction between the meson and the quark-antiquark pair,

$$
\Gamma^{\mu}=N \frac{\gamma^{\mu}-\left(k_{q}+k_{\bar{q}}\right)^{\mu} /\left(M_{i, f}+2 m_{q}\right)}{\left[k_{q}^{2}-m_{R}^{2}+\imath \epsilon\right]\left[k_{\bar{q}}^{2}-m_{R}^{2}+\imath \epsilon\right]},
$$

where, for the $u$ quark contribution, the struck $u$ quark momentum is $k_{u}=k-\Delta / 2$ and the spectator constituent momentum is $k_{s}=k_{\bar{d}}=k-P . N$ is the normalization constant, $m_{q}$ and $m_{R}$ are the masses of the constituent quark and the regulator, respectively. $M_{i, f}$ are the kinematic invariant masses with subscript $i$ for initial vertex and $f$ for the final vertex,

$$
\begin{gathered}
M_{i}^{2}=\frac{\kappa_{\perp}^{2}+m_{q}^{2}}{1-x^{\prime}}+\frac{\kappa_{\perp}^{2}+m_{q}^{2}}{x^{\prime}}, \\
M_{f}^{2}=\frac{\kappa_{\perp}^{\prime 2}+m_{q}^{2}}{1-x^{\prime \prime}}+\frac{\kappa_{\perp}^{\prime 2}+m_{q}^{2}}{x^{\prime \prime}},
\end{gathered}
$$

with the light-front momentum fractions $x^{\prime}=-k_{s}^{+} / p^{+}=$ $(1-x) /(1-|\xi|), x^{\prime \prime}=x^{\prime} p^{+} / p^{+}=(1-x) /(1+|\xi|)$, and

$$
\begin{gathered}
\kappa_{\perp}=k_{s \perp}-\frac{k_{s}^{+}}{p^{+}} p_{i \perp}=(k-P)_{\perp}-\frac{x^{\prime}}{2} \Delta_{\perp}, \\
\kappa_{\perp}^{\prime}=(k-P)_{\perp}+\frac{x^{\prime \prime}}{2} \Delta_{\perp} .
\end{gathered}
$$

In the Efremov-Radyushkin-Brodsky-Lepage (ERBL) regime (i.e., nonvalence regime), the relation of $-|\xi|<$ $x<|\xi|$ leads to $x^{\prime}>1$, and the initial vertex becomes the nonwave-function vertex which means $M_{i}^{2}$ can get negative values. To keep the mass square positive, we follow Ref. [40] by directly replacing $1-x^{\prime}$ with $x^{\prime}-1$ in Eq. (20a) and gets

$$
\tilde{M}_{i}^{2}=\frac{\kappa_{\perp}^{2}+m_{q}^{2}}{x^{\prime}-1}+\frac{\kappa_{\perp}^{2}+m_{q}^{2}}{x^{\prime}} .
$$


When both the struck and spectator constituents are on mass shells, one gets $M_{i}^{2}=M_{f}^{2}=m^{2}$ but $\tilde{M}_{i}^{2} \neq m^{2}$. The physics in the ERBL regime is much more complicated than that in the Dokshitzer-Gribov-Lipatov-Altarelli-Parisi one, since the creation of the $q \bar{q}$ pair involves an infinite sum of the meson contribution. The above simple method may omits the rich details. As Eq. (16) shows, the GFFs $D_{0}(t)$ and $D_{1}(t)$ is bound to $\xi$ and the nonzero $\xi$ requires

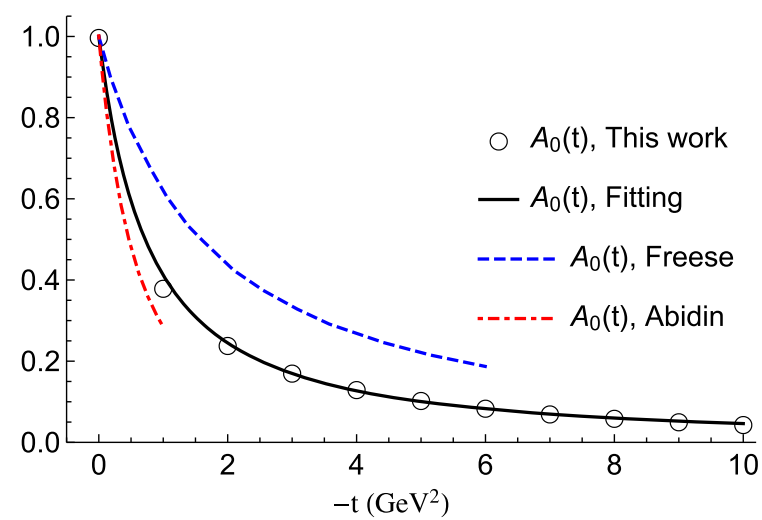

(a)

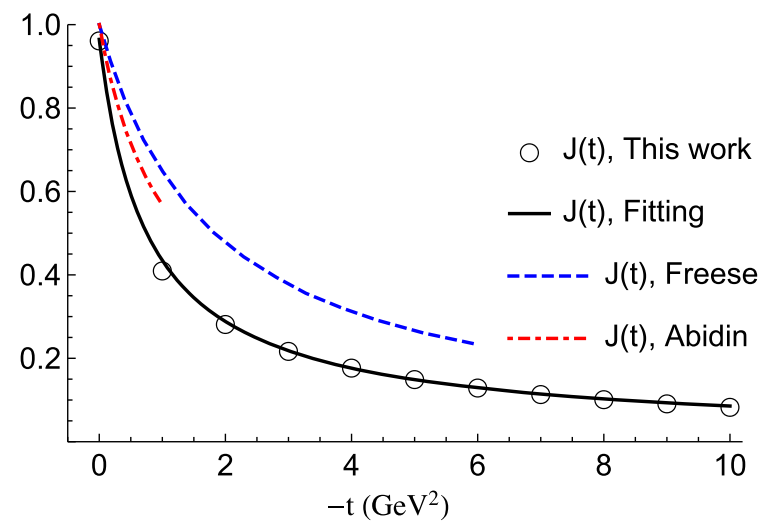

(c)

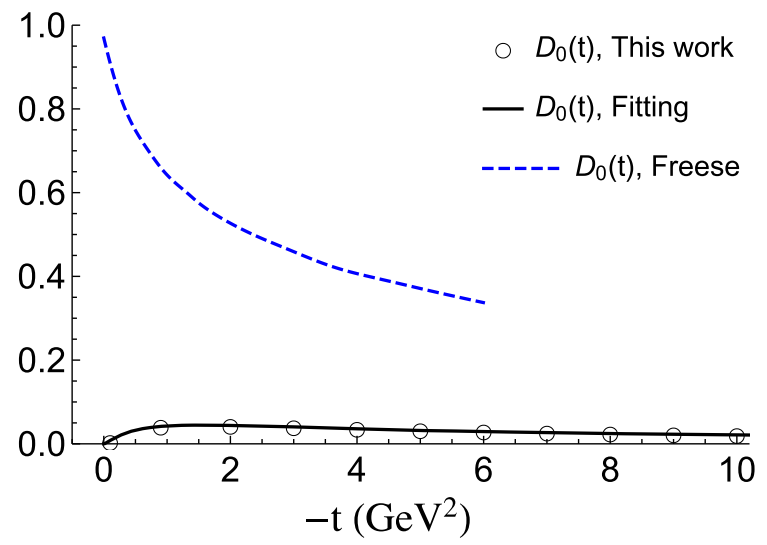

(e) the ERBL regime. It is one possible reason why our results for these two GFFs are very different with that of other models and the free theory.

\section{NUMERICAL RESULTS}

Following our previous work on the $\rho$ meson GPDs [3234], we take the two model parameters, the constituent

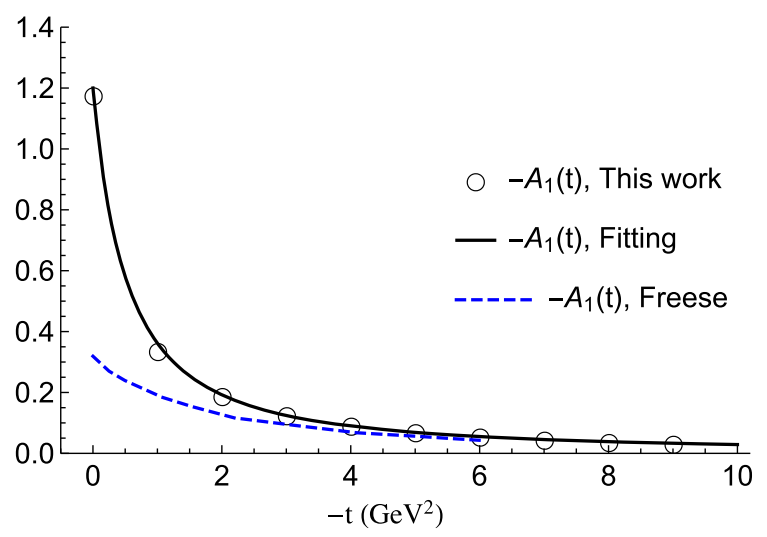

(b)

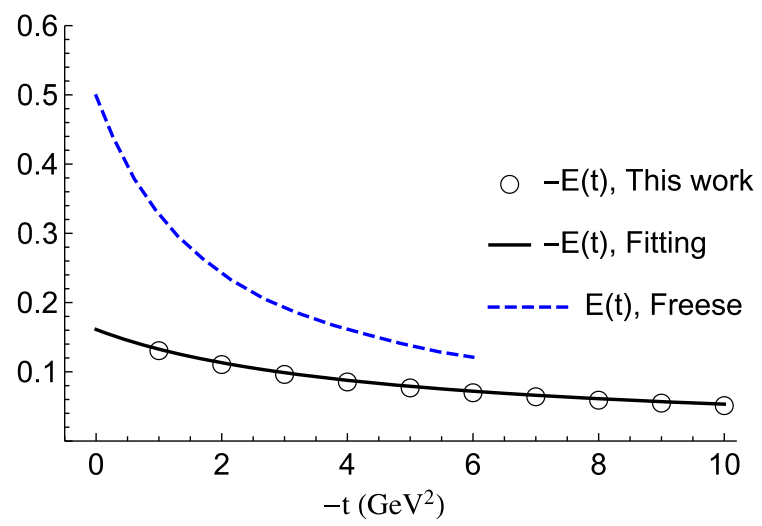

(d)

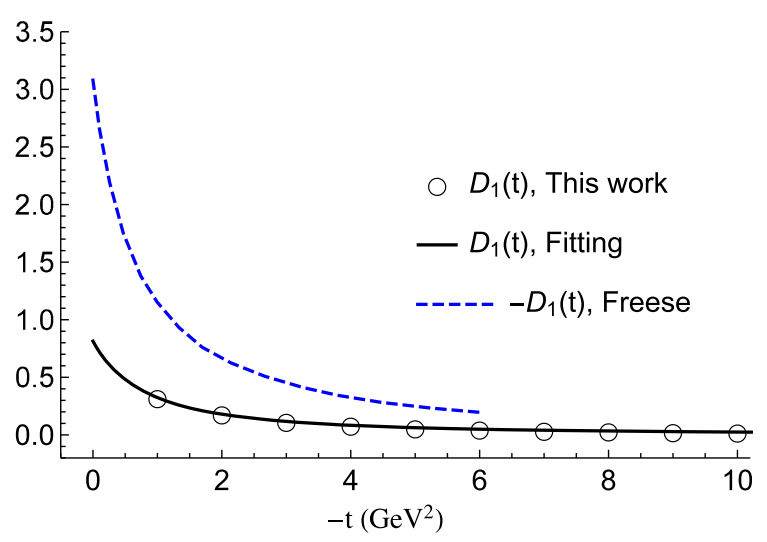

(f)

FIG. 2. Gravitational form factors (a) $A_{0}(t), A_{1}(t)$, (b) $J(t), E(t)$, (c) $D_{0}(t)$, and (d) $D_{1}(t)$. The solid lines are parametric fittings and the empty circles are model results. The red dot-dashed lines are results from the AdS/QCD approach by the Abidin etc. [30] and the blue dashed lines are results from the NJL model by Freese etc. [31]. To compare the results from Ref. [30] with ours, we need take the scale $\Lambda_{\mathrm{QCD}}=0.226 \mathrm{GeV}$ which is employed in our previous work [32]. 
mass $m_{q}=0.403 \mathrm{GeV}$ and regulator mass $m_{R}=$ $1.61 \mathrm{GeV}$. The renormalization scale is about $0.5 \mathrm{GeV}$. In our LCCQM, the gluon contributions are assumed to be absorbed into the constituent quark mass. After summing over all the contributions from the quark flavors, we get the total GFFs, where the 3 energy-momentum nonconserving terms are canceled, and only 6 conserving terms are considered.

Our results for GFFs are shown in Fig. 2. We know that the GFFs $A_{0}(t)$ and $J(t)$ are related to the generators of the Poincare group for the mass and spin of the particle (here is the $\rho$ meson) which give the constraints at zero-momentum transfer $A_{0}(0)=1$ and $J(0)=1[26,30,41,42]$. Except for the cases of free particles and Goldstone bosons, there is no any other general principles or constraints for $D$-term [4]. The results for the $D$-terms of the proton and pion have been given by different phenomenological analyses based on the experimental data of leading order deep virtual Compton scattering (DVCS) process [43]. In the spin-1 case, the $D$-term is related to the GFFs $D(t)$ and $E(t)$. It is expected, with a similar approach, one can also obtain an estimation for the mechanical properties for other spin-1 particles, in particular for deuteron, which may be measured in the future JLab experiment [44].

In the literature, there are some other model calculations for the spin-1 GFFs before present work. Reference [30] gives the results for $A_{0}(t)$ and $J(t)$ by applying the AdS/ QCD approach, and Ref. [31] shows the six nonzero GFFs in the NJL model. The relations among the different notations have been explicitly discussed in Ref. [27]. Besides the common constraints from the total mass $\left(A_{0}(0)=1\right)$ and spin $(J(0)=1)$, the present calculation and the mentioned two other approaches, however, show different decreasing $t$-dependent behaviors of the two GFFs, $A_{0}(t)$ and $J(t)$. For the rest four nonzero GFFs $A_{1}$, $D_{0}, D_{1}$, and $E$, our results are also quite different from that in the NJL model [31]. One could find, in the common region of momentum transfer, the absolute value of our $A_{1}(t)(\sim 1.2$ at $t=0)$ is much larger than the results ( $\sim 0.4$ at $t=0)$ in Ref. [31] and the absolute value of our $E(t)$ $(\sim 0.15$ at $t=0)$ is smaller than that $(\sim 0.5$ at $t=0)$ in Ref. [31]. For $D_{0}(t)$, we get $D_{0}(0) \sim 0$ which is a significant difference w.r.t. the free theory $D_{0}(0)=1$ (without the nonminimal term) [27] and the chiral limit [31]. One possible reason for such a difference is due to the simplification we used in the model, as discussed previously. The other possible reason is that the quark mass is large and much away from the chiral limit. For $D_{1}(t)$ we get opposite sign comparing with that from Ref. [31].

The present model result for $\rho$ meson D-term is

$$
\begin{aligned}
D & =\mathcal{D}_{0}(0)=-D_{0}(0)+\frac{4}{3} E(0) \\
& =0+\frac{4}{3} \cdot(-0.161)=-0.21<0 .
\end{aligned}
$$

It should be stressed that the negative value of the $D$-term satisfies the requirement for the mechanical stability [4], i.e.,

$$
\frac{2}{3} s_{0}(r)+p_{0}(r)>0
$$

Although the t-dependent behaviors of GFFs obtained in different approaches are quite different, we find the $D$-term value obtained from the GFFs of Ref. [31] (although it is not given explicitly in the paper) is around -0.33 , which is close to ours -0.21 .

It is shown that the 3D Fourier transforms in the Breit frame (BF) and two-dimensional(2D) Fourier transforms in the Light-Cone (LC) frame gives different definitions of the mass radii $\left\langle r^{2}\right\rangle_{\text {mass }}[5,31,45]$. In the Breit frame, we have ${ }^{2}$

$$
\begin{aligned}
\left\langle r^{2}\right\rangle_{\mathrm{BF}} & =\frac{\int d^{3} r r^{2} T^{00}(\vec{r})}{\int d^{3} r T^{00}(\vec{r})}=\frac{1}{m} \int d^{3} \mathbf{r} r^{2} T^{00}(\vec{r}) \\
& =\left.6 \frac{d A_{0}(t)}{d t}\right|_{t \rightarrow 0}+\frac{1}{m^{2}}\left[-\frac{7}{4} A_{0}(0)+\frac{1}{2} A_{1}(0)+\frac{3}{2} D_{0}(0)+2 J(0)-E(0)\right]
\end{aligned}
$$

Eq. (25) is equivalent to Eq. (36) of Ref. [28]. In the light-cone frame, it is obtained in Ref. [31] as

$$
\left\langle r^{2}\right\rangle_{\mathrm{LC}}=\left.4 \frac{d A_{0}(t)}{d t}\right|_{t=0}+\frac{1}{3 m^{2}}\left[2 A_{0}(0)+A_{1}(0)-2 J(0)+2 E(0)\right]
$$

\footnotetext{
${ }^{2}$ In our previous proceeding paper [46], the definition of $\left\langle r^{2}\right\rangle_{\text {mass }}$ in its Eq. (7) is wrong. In Ref. [30] where an AdS/QCD model calculation is preformed, its Eq. (46) defines the radius as $\left\langle r^{2}\right\rangle_{\text {mass }}=-\left.6 \frac{\partial A}{\partial Q^{2}}\right|_{Q^{2}=0}$ with $Q^{2}=-t$.
} 
TABLE I. Mean squared mass radius, mass and quadrupole moment of $\rho$ meson by this work, the NJL model [31] and the AdS/QCD model [30], respectively. All radii are in fm, the mass quadrupole moment is in units of $m_{\rho}-\mathrm{fm}^{2}$, and the electric quadrupole moment is in $e$-fm. In Ref. [30], it is not specified in which frame the definition of radii is given, and the mass definition differ from that the Breit frame and light cone prescriptions used in the present work and Ref. [31].

\begin{tabular}{lcccc}
\hline \hline & $\sqrt{\left\langle r^{2}\right\rangle_{\text {mass }}}$ & $\sqrt{\left\langle r^{2}\right\rangle_{\text {elec }}}$ & $\mathcal{Q}_{\text {mass }}$ & $\mathcal{Q}_{\text {elec }}$ \\
\hline AdS/QCD [30] & 0.46 & 0.73 & & \\
NJL [31], Briet frame & 0.45 & 0.67 & -0.0224 & -0.0200 \\
NJL [31], Light Cone & 0.32 & 0.45 & & \\
this work, Briet frame & 0.53 & 0.72 & -0.0322 & -0.0212 \\
this work, Light Cone & 0.41 & & & \\
\hline \hline
\end{tabular}

As discussed in Ref. [31,45], the 3D spatial distributions in the Breit frame are found not invariant under Lorentz boosts. While the relativistic corrections are intrinsically accounted for in the light-cone frame and therefore the physical meaning of the radius of $2 \mathrm{D}$ transverse distributions in light-cone is more clear. The numerical results of these radii are list in Table I in comparing with other model predictions. In the present work, we have $\sqrt{\left\langle r^{2}\right\rangle_{\mathrm{BF}}}=$ $0.53 \mathrm{fm}$ and $\sqrt{\left\langle r^{2}\right\rangle_{\mathrm{LC}}}=0.41 \mathrm{fm}$. In both frames, the mass radii are smaller than the charge radius $(0.72 \mathrm{fm})$ from our previous calculation. This feature is reasonable and consistent with the nucleon case $[25,47]$. Besides, we find both of the radius and the $D$-term are sensitive to the constituent quark mass $m_{q}$. As binding energy is approaching zero $\left(m_{q}\right.$ is approaching to the half of total $\rho$ meson mass), the radius increases rapidly and the absolute value of $D$-term decreases close to zero. It means the bound system is getting looser or even falls apart in the view of the constituent quark model. It is consistent with the observation in Ref. [48] that the value of $D$-term vanishes in the free Dirac fermions case.

The gravitational quadrupole moment,

$$
\begin{aligned}
\mathcal{Q}_{\text {mass }} & =-\frac{1}{m}\left[-A_{0}(0)+\frac{1}{2} A_{1}(0)+2 J(0)-E(0)\right] \\
& =-0.0322\left[m_{\rho}-\mathrm{fm}^{2}\right],
\end{aligned}
$$

in the present model. It is consistent with that from the NJL model prediction $\left(-0.0224\left[m_{\rho}-\mathrm{fm}^{2}\right]\right)$ in Ref. [31]. The quadrupole moments of mass and charge are close under the comparable units. The same (negative) sign implies the mass and charge distributions are synchronous when the particle becomes polarized.

In principle, one can calculate the static EMT $T^{\mu \nu}(r)$ (also the energy density and pressure) straightforwardly from the obtained GFFs with Fourier transformation. However, the integrals may not converge if the GFFs drops slowly with respect to the momentum transfer square $t$. According to the analyses of $\mathrm{pQCD}$ and AdS/CFT, at the large momentum transfer $\left(-t=Q^{2} \rightarrow \infty\right)$, the six GFFs decease roughly with the following power respectively [30],

$$
\left(A_{0}, D_{0}, J, E\right) \sim 1 / t^{2}, \quad\left(A_{1}, D_{1}\right) \sim 1 / t^{3} .
$$

In the nucleon case, Ref. [49] adopts an assumption that its GFFs behave $d_{1}(t) \sim t^{-3}$, and the converged results are obtained.

Because of the limited capability of our LCCQM, especially at the large momentum transfer region, we believe that a modification of our model results in the large momentum transfer region is needed. To simulate the $t$-dependent behaviors of the obtained GFFs, we consider the forms like

$$
a\left(1-\frac{t}{b}\right)^{c}
$$

to present our numerical results at a momentum transfer region, $0<-t<10 \mathrm{GeV}^{2}$, and we find five of the six GFFs are approximately described by

$$
\begin{gathered}
A_{0}(t)=(1-0.996 t)^{-1.28}, \\
A_{1}(t)=-1.20\left(1-\frac{t}{0.73}\right)^{-1.38}, \\
D_{1}(t)=0.814\left(1-\frac{t}{1.32}\right)^{-1.64}, \\
J(t)=0.965\left(1-\frac{t}{0.68}\right)^{-0.877}, \\
E(t)=-0.161\left(1-\frac{t}{4.2}\right)^{-0.909} .
\end{gathered}
$$

Due to the limit $|\xi| \leq 1 / \sqrt{1-4 M^{2} / t}$, there are some small oscillation in the numerical result of the GFF $D_{0}(t)$ at $0<-t<3 \mathrm{GeV}^{2}$. After a careful check of its $t-$ dependent behavior, we find it is oscillating around a curve that can be simulated by

$$
\left(1-\frac{t}{b}\right)^{c}\left(1-\frac{d}{t}\right)^{e}
$$

Thus, for $D_{0}(t)$, we get

$$
D_{0}(t)=\left(1-\frac{0.97}{t}\right)^{-1.97}\left(1-\frac{t}{0.14}\right)^{-0.86}
$$

It turns out, unlike the nucleon case in Ref. [49], neither our GFFs results in Eqs. (30) and (32) nor the pQCD predictions in Eq. (28) drop fast enough to give converging 
results for $T^{\mu \nu}(r)$ [see Eq. (2)]. This issue has already been pointed out and discussed for the cases of pion meson [22] and nucleon [5]. One possible reason is that the integrals that defining $T^{\mu \nu}(r)$ is subjected to the relativistic corrections. For the spin-0 case, Ref. [22] estimated the relativistic corrections by a way of smearing out the pointparticles (a delta function is replaced by a Gaussian function). It is believed that the relativistic corrections $\left(\delta_{\text {rel }} \equiv 1 /\left(2 m^{2} R_{h}^{2}\right)\right)$ is negligible when $m R_{h} \gg 1$ where $m$ is the hadron mass and $R_{h}$ is the hadron size. For the light meson pion, its $\delta_{\text {rel }}=220 \%$ [22]. Although the $\rho$ meson is spin-1 hadron, we may simply "borrow" the argument for pion to roughly estimate how large the relativistic corrections are for the case of the $\rho$ meson. With our model estimation of the radius $\left(\sqrt{\langle r\rangle_{\text {grav }}^{2}} \sim 0.53 \mathrm{fm}\right)$, one gets $\delta_{\text {rel }} \sim 12 \%$ which is not important and we believe that the concept of the 3D densities is applicable for the $\rho$ meson. So far, there is no experimental data for the $\rho$ meson radius, the future experimental information about its size would be essential for our estimate.

To proceed with a modification of our phenomenological model calculation, particularly in the large $t$ region, we introduce a Gaussian form wave package, as we did in previous work [33], to suppress the contribution from the high energy region. It's reasonable since only limited values of $t$ can be measured in the experiments. Choosing the Gaussian form wave package originates from the observation that a hadron is an extended object and is smeared out in space [50]. It should be stressed that the value of $D$-term is not affected by this modification and by the consideration of the relativistic corrections since it's defined by the value of GFFs at the zero momentum transfer $[4,22]$.

In our previous study for the $\rho$-meson transverse distributions in the 2D impact parameter space [33], we introduced a 2D Gaussian form wave packet in both of the incoming and outgoing states in order to avoid the

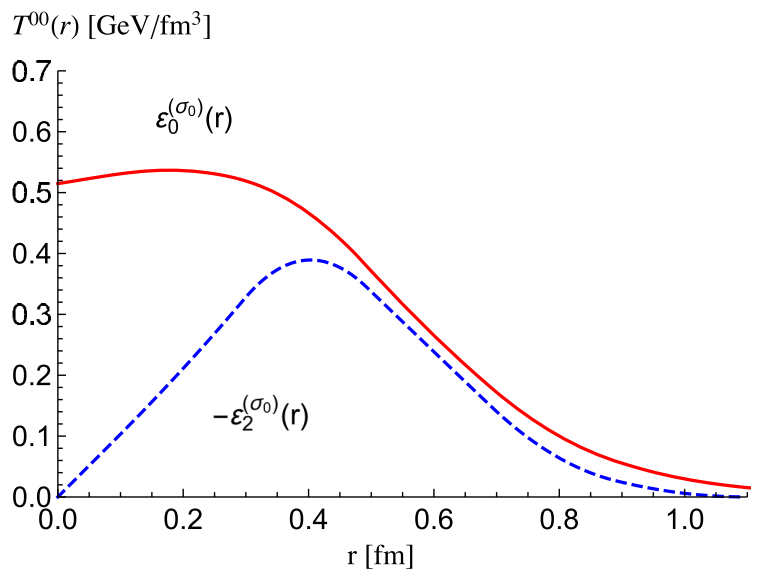

(a) similar divergences, and we found the appropriate value for the wave packet width being around $\sigma=1-2 \mathrm{GeV}^{-1}$. This treatment was also pointed out and employed in other calculations of nucleon GPDs in the impact parameter space [50]. Here, a similar 3D Gaussian form wave packet,

$$
e^{-\vec{\Delta}^{2} \sigma_{0}^{2} / 4}
$$

is adopted (with width $\sigma_{0} \sim 2 \mathrm{GeV}^{-1}=0.39 \mathrm{fm}$ ) to carry out the calculation for the spatial distributions. As a result, the expressions for the energy densities are modified to be

$$
\begin{gathered}
\varepsilon_{0}^{\left(\sigma_{0}\right)}(r)=2 m^{2} \int \frac{d^{3} \Delta}{2 E(2 \pi)^{3}} e^{-i \vec{\Delta} \cdot \vec{r}-\vec{\Delta}^{2} \sigma_{0}^{2} / 4} \mathcal{E}_{0}(t), \\
\varepsilon_{2}^{\left(\sigma_{0}\right)}(r)=-r \frac{d}{d r} \frac{1}{r} \frac{d}{d r} \int \frac{d^{3} \Delta}{2 E(2 \pi)^{3}} e^{-i \vec{\Delta} \cdot \vec{r}-\vec{\Delta}^{2} \sigma_{0}^{2} / 4} \mathcal{E}_{2}(t) .
\end{gathered}
$$

Then, the spin distribution is modified to be

$$
\begin{aligned}
J_{a}^{i\left(\sigma_{0}\right)}\left(r, \sigma^{\prime}, \sigma\right)= & J_{a}^{i\left(\sigma_{0}\right)}\left(\vec{r}, \sigma^{\prime}, \sigma\right) \\
= & \hat{S}_{\sigma^{\prime} \sigma}^{j} \int \frac{d^{3} \Delta}{(2 \pi)^{3}} e^{-i \vec{\Delta} \cdot \vec{r}-\vec{\Delta}^{2} \sigma_{0}^{2} / 4} \\
& \times\left[\left(\hat{\mathcal{J}}^{a}(t)+\frac{2}{3} t \frac{d \hat{\mathcal{J}}^{a}(t)}{d t}\right) \delta^{i j}\right. \\
& \left.+\left(\Delta^{i} \Delta^{j}-\frac{1}{3} \vec{\Delta}^{2} \delta^{i j}\right) \frac{d \hat{\mathcal{J}}^{a}(t)}{d t}\right],
\end{aligned}
$$

where $\hat{\mathcal{J}}^{a}(t)=\frac{m}{E} \mathcal{J}^{a}(t)$ and the first step in the above equation is based on the observation that $J_{a}^{i}$ depends on $\vec{r}$ only through $r=|\vec{r}|$. Moreover, the function $\tilde{\mathcal{D}}_{n}(r)$ in Eq. (15a), which defines the distributions of pressure and shear force, is modified as

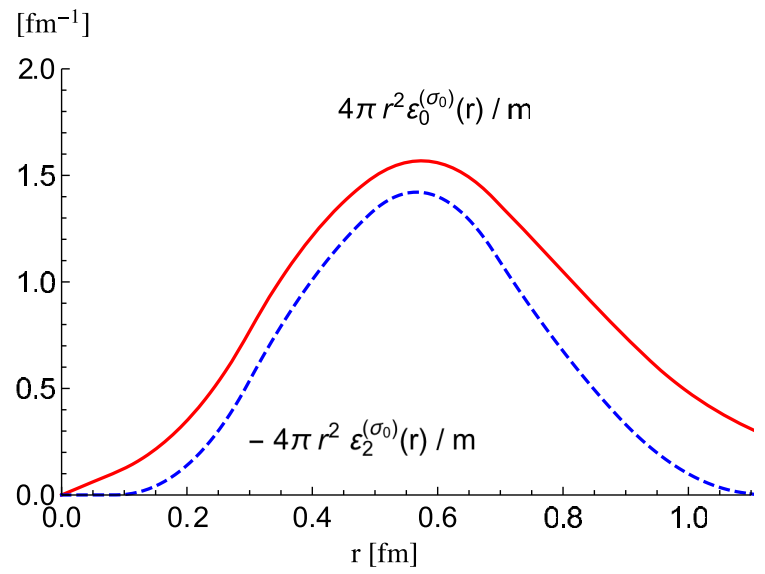

(b)

FIG. 3. Energy densities (a) $\varepsilon_{0}^{\left(\sigma_{0}\right)}(r)$, (b) $\varepsilon_{2}^{\left(\sigma_{0}\right)}(r)$ with $\sigma_{0}=2 \mathrm{GeV}^{-1}$. 


$$
\begin{aligned}
\tilde{\mathcal{D}}_{0}^{\left(\sigma_{0}\right)}(r)= & 2 m \int \frac{d^{3} \Delta}{2 E(2 \pi)^{3}} e^{-i \vec{\Delta} \cdot \vec{r}-\vec{\Delta}^{2} \sigma_{0}^{2} / 4} \mathcal{D}_{0}(t), \\
\tilde{\mathcal{D}}_{2}^{\left(\sigma_{0}\right)}(r)= & 2 m \int \frac{d^{3} \Delta}{2 E(2 \pi)^{3}} e^{-i \vec{\Delta} \cdot \vec{r}-\vec{\Delta}^{2} \sigma_{0}^{2} / 4} \mathcal{D}_{2}(t) \\
& +\frac{2}{m}\left(\frac{d}{d r} \frac{d}{d r}-\frac{2}{r} \frac{d}{d r}\right) \\
& \times \int \frac{d^{3} \Delta}{2 E(2 \pi)^{3}} e^{-i \vec{\Delta} \cdot \vec{r}-\vec{\Delta}^{2} \sigma_{0}^{2} / 4} \mathcal{D}_{3}(t), \\
\tilde{\mathcal{D}}_{3}^{\left(\sigma_{0}\right)}(r)= & -\frac{4}{m}\left(\frac{d}{d r} \frac{d}{d r}-\frac{2}{r} \frac{d}{d r}\right) \\
& \times \int \frac{d^{3} \Delta}{2 E(2 \pi)^{3}} e^{-i \vec{\Delta} \cdot \vec{r}-\vec{\Delta}^{2} \sigma_{0}^{2} / 4} \mathcal{D}_{3}(t) .
\end{aligned}
$$

Correspondingly, $p_{n}(r) \rightarrow p_{n}^{\left(\sigma_{0}\right)}(r)$ and $s_{n}(r) \rightarrow s_{n}^{\left(\sigma_{0}\right)}(r)$. After summing over all the partons (only quarks here) in Eq. (35), one has

$$
J_{\sigma^{\prime} \sigma}^{i\left(\sigma_{0}\right)}(\vec{r})=\sum_{a} J_{a}^{i\left(\sigma_{0}\right)}\left(\vec{r}, \sigma^{\prime}, \sigma\right), \quad i, \sigma^{\prime}, \sigma=x, y, z
$$

In the Cartesian basis, average over all polarizations and spatial directions, one further has

$$
J^{\left(\sigma_{0}\right)}(r) \equiv \frac{1}{\operatorname{Tr}\left[\hat{\mathbf{S}}^{2}\right]} \sum_{\sigma^{\prime} \sigma i} \hat{S}_{\sigma^{\prime} \sigma}^{i} J_{\sigma^{\prime} \sigma}^{i\left(\sigma_{0}\right)}(\vec{r})=i J_{y z}^{x\left(\sigma_{0}\right)}(r),
$$

which is a real quantity and is the 3D spatial spin distribution of the $\rho$ meson.

With those preparations, the energy densities calculated from the GFFs in Eqs. (30) and (32) are shown in Fig. 3. In Fig. 3(b), the normalization is changed to the form of $4 \pi r^{2} \varepsilon_{0}^{\left(\sigma_{0}\right)}(r) / m$, which gives 1 after averaging over the polarizations and integrating over the whole radial space. The higher-order term $\varepsilon_{2}^{\left(\sigma_{0}\right)}(r)$ does not contribute to the energy distributions in the unpolarized case. Its negative value indicates that the mass or energy distribution would deviate from the center because of the polarization effect. As shown in Table I, the values of charge and mass quadrupole moments in our work and Ref. [31] are all negative. In the classical picture, a negative quadrupole moment corresponding to an oblate ellipsoid distribution. Thus the charge and mass distributions are consistent in shape.

The result for the spin distribution is shown in Fig. 4. In our previous work with LCCQM, we obtain the fraction of spin carried by the constituent quark and antiquark in $\rho$ meson is $86 \%$ [34]. The rest part is believed to come from the orbital angular momentum (no gluon in our model) [51-54].

Finally, our results for pressures $p_{n}^{\left(\sigma_{0}\right)}$ and shear forces $s_{n}^{\left(\sigma_{0}\right)}$ are shown in Fig. 5. To the best of our knowledge, there are some model calculations for spin- 0 and spin- $1 / 2$

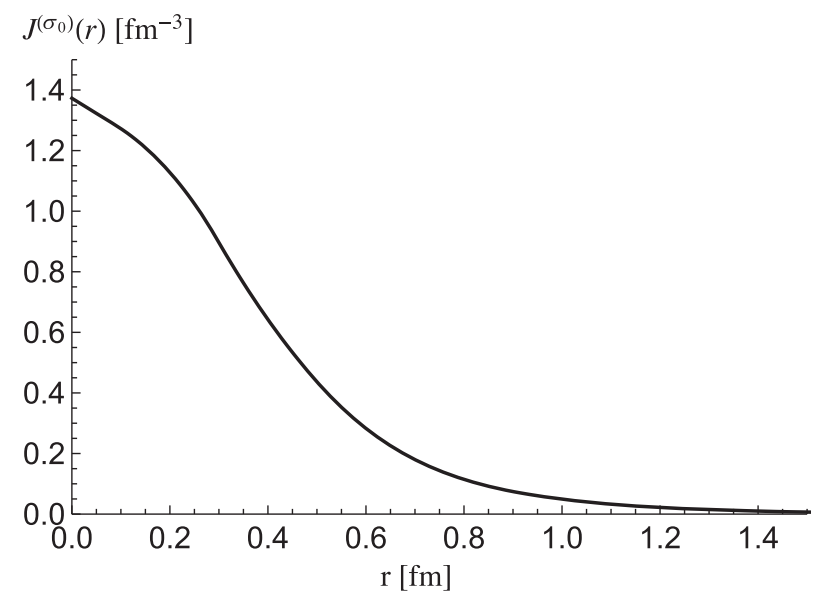

FIG. 4. Spin densities $J^{\left(\sigma_{0}\right)}(r)$ with $\sigma_{0}=2 \mathrm{GeV}^{-1}$.

nucleon, but none for the spin-1 particle before. In the unpolarized case, only the first order pressure and shear force contribute to $T^{i j}$ in Eq. (12) and the normal force $d F_{r}$ in Eq. (B1). So far in almost all model studies for different spin cases, it is found that the (unpolarized) pressure is positive in the inner region and negative in the outer region. Under the present convention, we know that the positive sign means repulsion toward outside and the negative sign means attraction toward inside. However, the specific relationship between these values and the strong force remains obscure. As one can see, $p_{0}^{\left(\sigma_{0}\right)}(r)$ changes its sign for the first time at around $r \sim 0.5 \mathrm{fm}$, which is roughly the gravitational radius. Physically, the sign-changing means the forces change from "stretching" to "squeezing." This phenomenon is the same as in the nucleon case [4]. In the polarized case, the higher-ordered pressures $p_{2}(r), p_{3}(r)$ and shear forces $s_{2}(r), s_{3}(r)$, contributes to both normal and tangential forces, as shown in Eq. (B1) and (B2). The size of tangential forces are proportional to $p_{2}(r)+\frac{2}{3} s_{2}(r)$ which keeps negative as shown in Fig. 5(c). The final sign of $d F_{\theta}$ and $d F_{\phi}$ is, however, also dependent on the spherical quadrupole tensor elements as shown in Appendix B. In the large $-N_{c}$ limit with the baryon as chiral soliton, it is found that $p_{2}(r)=s_{2}(r)=0$ for the $\Delta$ baryon $(J=3 / 2)$ [35], which is a very interesting prediction. At the region, $r \geq 1 \mathrm{fm}$, the pressures and shear forces are all quickly approaching to zero with small oscillations with respect to the center region values. There always exist oscillations in this approach and the oscillations depend on what the value of $\sigma_{0}$ is used in our numerical calculation (larger $\sigma_{0}$ generates stronger suppression on the amplitudes of the oscillations). There is still no constraint on how many times the changing would happen and no explanation for the meaning of those numbers. It is of great interest to have further study and to answer these intriguing questions. Nevertheless, here we present the first model estimation for the pressures and shear forces for the spin- $1 \rho$ meson. Similar distributions 


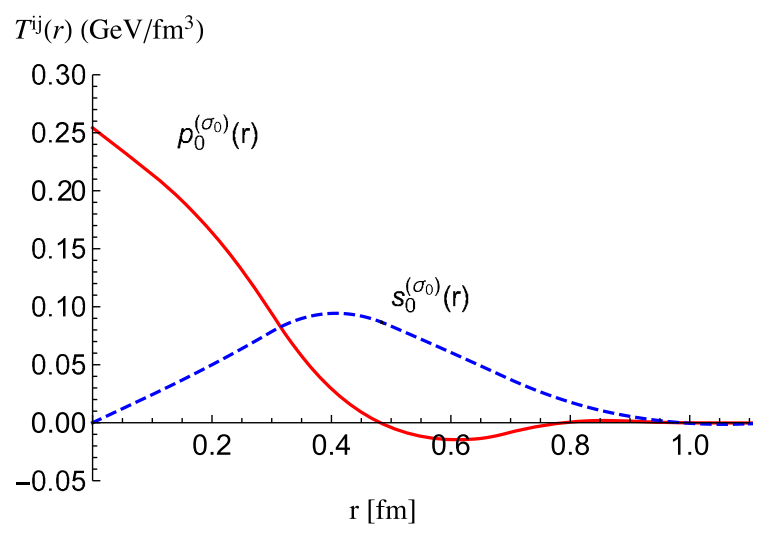

(a)

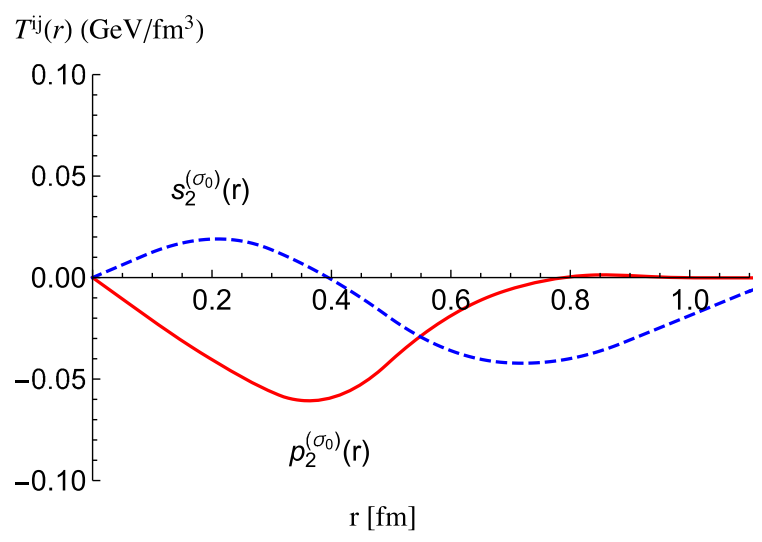

(c)

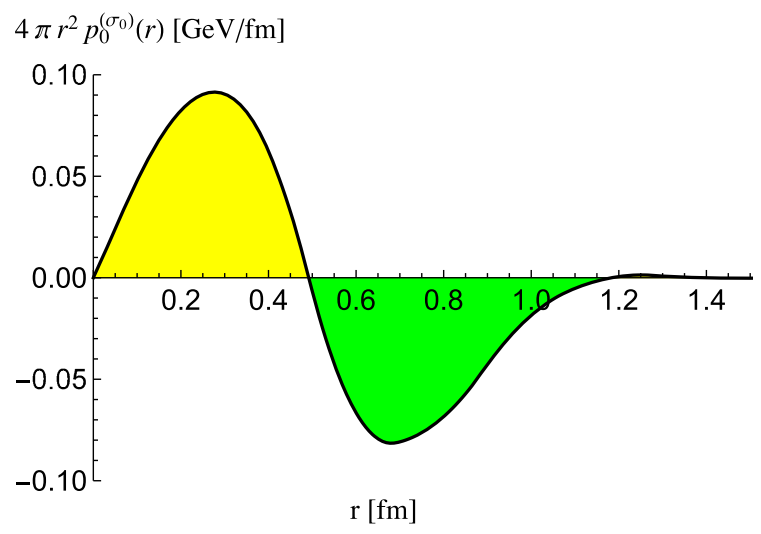

(b)

$T^{\mathrm{ij}}(r)\left(\mathrm{GeV} / \mathrm{fm}^{3}\right)$

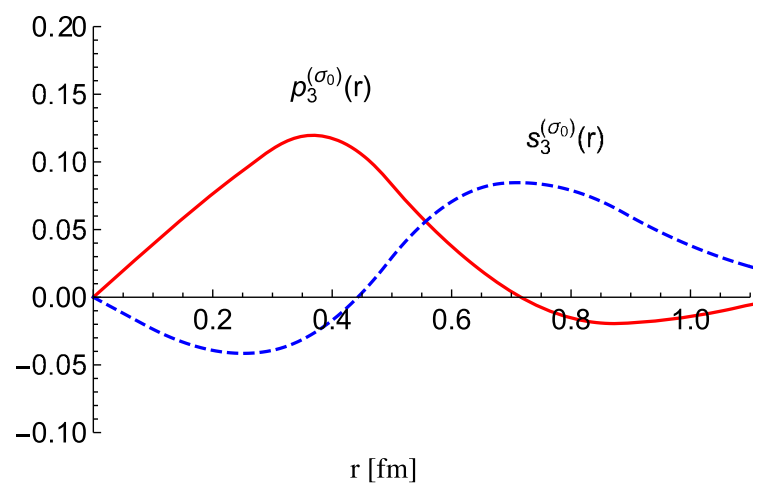

(d)

FIG. 5. The pressure and shear forces functions with $\sigma_{0}=2 \mathrm{GeV}^{-1}$.

may indicate some common properties of the strong force in forming the hadron systems.

\section{SUMMARY}

In this work, we extend our previous approach on the $\rho$ meson GPDs with the phenomenological light-front constituent quark model to its GFFs and further to the distributions of pressure and shear forces. For the GFFs $A_{0}$ and $J$ which are related to the mass and spin, our model estimations are consistent with the result of other approaches, such as the NJL model and AdS/CFT etc.. For the rest four GFFs, there are no specific constraints as the mass and spin cases, and the results from different approaches have large discrepancies even with opposite signs. Moreover, the $D$-term is given through our calculated GFFs, and it is estimated to be -0.21 . The negative value satisfies the stability condition. We also calculate the distributions of energy, pressure, spin, and shear forces. The results for the mass radius and quadrupole moment also agree with previous calculations in the NJL model and the AdS/QCD model etc. Since the LCCQM works well mainly within the low momentum transfer regions, we consider a Gaussian wave package during the Fourier transforms to suppress the contributions from large momentum transfer regions. Thus, the present results should be considered as a qualitative estimation. We expect that our results may provide some hints for the understanding of the mechanical properties, especially in the case of spin-1 hadron.

\section{ACKNOWLEDGMENTS}

We acknowledge helpful conversations with Maxim V. Polyakov and Julia Yu. Panteleeva. This work is supported by the National Natural Sciences Foundations of China under the Grants No. 11975245, No. 11521505, No. 11565007, No. 11635009, and No. 11947228, the Sino-German CRC 110 by NSFC under Grant No. 11621131001, the Key Research Program of CAS, Grant No. Y7292610K1, the IHEP Innovation Fund No. Y4545190Y2 and China Postdoctoral Science Foundation under Grant No. 2019M662316. 


\section{APPENDIX A: DEFINITION FOR THE FORM FACTORS}

The definitions for the form factors used in this work are

$$
\begin{gathered}
\mathcal{E}_{0}^{a}(t)=A_{0}^{a}(t)+\frac{1}{4} \bar{f}^{a}(t)-\frac{1}{2} \bar{c}_{0}^{a}(t) \\
+\frac{t}{12 m^{2}}\left[-5 A_{0}^{a}(t)+3 D_{0}^{a}(t)+4 J^{a}(t)-2 E^{a}(t)+A_{1}^{a}(t)+\frac{1}{2} \bar{f}^{a}(t)+\bar{c}_{0}^{a}(t)+\frac{1}{2} \bar{c}_{1}^{a}(t)\right] \\
-\frac{t^{2}}{24 m^{4}}\left[-A_{0}^{a}(t)+D_{0}^{a}(t)+2 J^{a}(t)-2 E^{a}(t)+A_{1}^{a}(t)+\frac{1}{2} D_{1}^{a}(t)+\frac{1}{4} \bar{c}_{1}^{a}(t)\right]+\frac{t^{3}}{192 m^{6}}\left[A_{1}^{a}(t)+D_{1}^{a}(t)\right], \\
\mathcal{E}_{2}^{a}(t)=-A_{0}^{a}(t)+2 J^{a}(t)-E^{a}(t)+\frac{1}{2} A_{1}^{a}(t)+\frac{1}{4} \bar{f}^{a}(t)+\frac{1}{2} \bar{c}_{0}^{a}(t)+\frac{1}{4} \bar{c}_{1}^{a}(t) \\
-\frac{t}{4 m^{2}}\left[-A_{0}^{a}(t)+D_{0}^{a}(t)+2 J^{a}(t)-2 E^{a}(t)+A_{1}^{a}(t)+\frac{1}{2} D_{1}^{a}(t)+\frac{1}{4} \bar{c}_{1}^{a}(t)\right] \\
+\frac{t^{2}}{32 m^{4}}\left[A_{1}^{a}(t)+D_{1}^{a}(t)\right], \\
\mathcal{J}^{a}(t)=J^{a}(t)+\frac{1}{2} \bar{f}^{a}(t)-\frac{t}{4 m^{2}}\left(J^{a}(t)-E^{a}(t)\right) . \\
\mathcal{D}_{0}^{a}(t)=-D_{0}^{a}(t)+\frac{4}{3} E^{a}(t)+\frac{t}{12 m^{2}}\left[2 D_{0}^{a}(t)-2 E^{a}(t)+D_{1}^{a}(t)\right]-\frac{t^{2}}{48 m^{4}} D_{1}^{a}(t), \\
\mathcal{D}_{2}^{a}(t)=-E^{a}(t), \\
\mathcal{D}_{3}^{a}(t)=\frac{1}{4}\left[2 D_{0}^{a}(t)-2 E^{a}(t)+D_{1}^{a}(t)\right]-\frac{t}{16 m^{2}} D_{1}^{a}(t) .
\end{gathered}
$$

When sum over all partons, the momentum-energy nonconserving terms, $\bar{f}^{a}$ and $\bar{c}_{0,1}^{a}$, will drop and they have no contribution.

\section{APPENDIX B: QUADRUPOLE TENSOR ELEMENTS}

The spherical components of the force acting on the infinitesimal radial area element $d S_{r}\left(d \boldsymbol{S}=d S_{r} \boldsymbol{e}_{r}+d S_{\theta} \boldsymbol{e}_{\theta}+d S_{\phi} \boldsymbol{e}_{\phi}\right)$ read [35]:

$$
\begin{aligned}
& \frac{d F_{r}}{d S_{r}}=p_{0}(r)+\frac{2}{3} s_{0}(r)+\hat{Q}^{r r}\left(p_{2}(r)+\frac{2}{3} s_{2}(r)+p_{3}(r)+\frac{2}{3} s_{3}(r)\right), \\
& \frac{d F_{\theta}}{d S_{r}}=\hat{Q}^{\theta r}\left(p_{2}(r)+\frac{2}{3} s_{2}(r)\right), \quad \frac{d F_{\phi}}{d S_{r}}=\hat{Q}^{\phi r}\left(p_{2}(r)+\frac{2}{3} s_{2}(r)\right) .
\end{aligned}
$$

Some of the spherical quadrupole tensor elements involved in Eq. (B1) and (B2) are,

$$
\begin{gathered}
\hat{Q}^{r r}=\left(\hat{Q}^{x x} \cos ^{2} \phi+\hat{Q}^{x y} \sin 2 \phi+\hat{Q}^{y y} \sin ^{2} \phi\right) \sin ^{2} \theta+\hat{Q}^{x z} \sin 2 \theta \cos \phi+\hat{Q}^{y z} \sin 2 \theta \sin \phi+\hat{Q}^{z z} \cos ^{2} \phi, \\
\hat{Q}^{\theta r}=\left(\hat{Q}^{x x} \cos ^{2} \phi+\hat{Q}^{x y} \sin 2 \phi+\hat{Q}^{y y} \sin ^{2} \phi-\hat{Q}^{z z}\right) \sin \theta \cos \theta+\left(\hat{Q}^{x z} \cos \phi+\hat{Q}^{y z} \sin \phi\right) \cos 2 \theta, \\
\hat{Q}^{\phi r}=\left(\left(\hat{Q}^{y y}-\hat{Q}^{x x}\right) \sin \phi \cos \phi+\hat{Q}^{x y} \cos 2 \phi\right) \sin \theta+\left(\hat{Q}^{y z} \cos \phi-\hat{Q}^{x z} \sin \phi\right) \cos \theta,
\end{gathered}
$$

where $\theta$ is the pole angle and $\phi$ is the azimuthal angle in commonly used polar coordinate system, and [36]

$$
\left(\hat{Q}^{i k}\right)_{l m}=\left(\hat{Q}_{i k}\right)_{l m}=-\frac{1}{2}\left(\delta_{i l} \delta_{k m}+\delta_{i m} \delta_{k l}-\frac{2}{3} \delta_{i k} \delta_{l m}\right), \quad(i, k, l, m=x, y, z) .
$$


[1] H. Pagels, Phys. Rev. 144, 1250 (1966).

[2] K. Goeke, M. V. Polyakov, and M. Vanderhaeghen, Prog. Part. Nucl. Phys. 47, 401 (2001).

[3] M. Polyakov, Phys. Lett. B 555, 57 (2003).

[4] M. V. Polyakov and P. Schweitzer, Int. J. Mod. Phys. A 33, 1830025 (2018).

[5] C. Lorcé, H. Moutarde, and A. P. Trawiński, Eur. Phys. J. C 79, 89 (2019).

[6] X.-D. Ji, Phys. Rev. Lett. 78, 610 (1997).

[7] M. V. Polyakov and C. Weiss, Phys. Rev. D 60, 114017 (1999).

[8] M. Diehl, Phys. Rep. 388, 41 (2003).

[9] A. V. Belitsky and A. V. Radyushkin, Phys. Rep. 418, 1 (2005).

[10] C.-R. Ji, Y. Mishchenko, and A. Radyushkin, Phys. Rev. D 73, 114013 (2006).

[11] T. Frederico, E. Pace, B. Pasquini, and G. Salme, Phys. Rev. D 80, 054021 (2009).

[12] M. Diehl, Eur. Phys. J. A 52, 149 (2016).

[13] B. Pasquini, S. Rodini, and A. Bacchetta, Phys. Rev. D 100, 054039 (2019).

[14] X.-D. Ji, Phys. Rev. Lett. 74, 1071 (1995).

[15] Y.-B. Yang, J. Liang, Y.-J. Bi, Y. Chen, T. Draper, K.-F. Liu, and Z. Liu, Phys. Rev. Lett. 121, 212001 (2018).

[16] D. Kharzeev, Proceedings of the International School of Physics "Enrico Fermi” 130, 105 (1996).

[17] D. Kharzeev, H. Satz, A. Syamtomov, and G. Zinovjev, Eur. Phys. J. C 9, 459 (1999).

[18] Y. Hatta, A. Rajan, and D.-L. Yang, Phys. Rev. D 100, 014032 (2019).

[19] W. Broniowski, E. Ruiz Arriola, and K. Golec-Biernat, Phys. Rev. D 77, 034023 (2008).

[20] W. Broniowski and E. R. Arriola, Phys. Rev. D 78, 094011 (2008).

[21] S. Kumano, Q.-T. Song, and O. Teryaev, Phys. Rev. D 97, 014020 (2018).

[22] J. Hudson and P. Schweitzer, Phys. Rev. D 96, 114013 (2017).

[23] C. Best, M. Gockeler, R. Horsley, E.-M. Ilgenfritz, H. Perlt, P. E. L. Rakow, A. Schafer, G. Schierholz, A. Schiller, and S. Schramm, Phys. Rev. D 56, 2743 (1997).

[24] I. V. Anikin, Particles 2, 357 (2019).

[25] K. Goeke, J. Grabis, J. Ossmann, M. V. Polyakov, P. Schweitzer, A. Silva, and D. Urbano, Phys. Rev. D 75, 094021 (2007).

[26] B. R. Holstein, Phys. Rev. D 74, 084030 (2006).
[27] M. V. Polyakov and B.-D. Sun, Phys. Rev. D 100, 036003 (2019).

[28] W. Cosyn, S. Cotogno, A. Freese, and C. Lorcé, Eur. Phys. J. C 79, 476 (2019).

[29] S. Cotogno, C. Lorcé, P. Lowdon, and M. Morales, Phys. Rev. D 101, 056016 (2020).

[30] Z. Abidin and C.E. Carlson, Phys. Rev. D 77, 095007 (2008).

[31] A. Freese and I. C. Cloët, Phys. Rev. C 100, 015201 (2019).

[32] B.-D. Sun and Y.-B. Dong, Phys. Rev. D 96, 036019 (2017).

[33] B.-D. Sun and Y.-B. Dong, Chin. Phys. C 42, 063104 (2018).

[34] B.-D. Sun and Y.-B. Dong, Phys. Rev. D 99, 016023 (2019).

[35] J. Y. Panteleeva and M. V. Polyakov, arXiv:2004.02912.

[36] D. A. Varshalovich, A. N. Moskalev, and V. K. Khersonsky, Quantum Theory of Angular Momentum: Irreducible Tensors, Spherical Harmonics, Vector Coupling Coefficients, 3nj Symbols (World Scientific, Singapore, 1988).

[37] C. Lorcé, L. Mantovani, and B. Pasquini, Phys. Lett. B 776, 38 (2018).

[38] M. V. Polyakov and P. Schweitzer, Proc. Sci., SPIN2018 (2019) 066 [arXiv:1812.06143].

[39] P. Schweitzer and K. Tezgin, Phys. Lett. B 796, 47 (2019).

[40] H.-M. Choi and C.-R. Ji, Phys. Rev. D 70, 053015 (2004).

[41] S. Cotogno, C. Lorcé, and P. Lowdon, Phys. Rev. D 100, 045003 (2019).

[42] C. Lorcé and P. Lowdon, Eur. Phys. J. C 80, 207 (2020).

[43] K. Kumerički and D. Müller, EPJ Web Conf. 112, 01012 (2016).

[44] M. Hattawy et al. (CLAS12 Run-Group), arXiv:1908 .00949 .

[45] G. A. Miller, Phys. Rev. C 99, 035202 (2019).

[46] B. Sun and Y. Dong, SciPost Phys. Proc. 3, 014 (2020).

[47] N. Bezginov, T. Valdez, M. Horbatsch, A. Marsman, A. C. Vutha, and E. A. Hessels, Science 365, 1007 (2019).

[48] J. Hudson and P. Schweitzer, Phys. Rev. D 97, 056003 (2018).

[49] V. D. Burkert, L. Elouadrhiri, and F. X. Girod, Nature (London) 557, 396 (2018).

[50] M. Diehl, Eur. Phys. J. C 25, 223 (2002); 31, 277(E) (2003).

[51] P. Hoodbhoy, X.-D. Ji, and W. Lu, Phys. Rev. D 59, 014013 (1998).

[52] C. Lorce and B. Pasquini, Phys. Rev. D 84, 014015 (2011).

[53] Y. Hatta, Phys. Lett. B 708, 186 (2012).

[54] K. Kanazawa, C. Lorcé, A. Metz, B. Pasquini, and M. Schlegel, Phys. Rev. D 90, 014028 (2014). 